\title{
SYMMETRY PROTECTED TOPOLOGICAL PHASES OF 3D BOSONS
}

(1) Physics of three dimensional bosonic topological insulators: Surface Deconfined Criticality and Quantized Magnetoelectric Effect.

Authors: Ashvin Vishwanath and T. Senthil, arXiv:1209.3058v2

(2) Three dimensional Symmetry Protected Topological Phase close to Antiferromagnetic Neel order

Author: Cenke Xu, arXiv:1209.4399

Recommended and a Commentary by M.P.A. Fisher, UC Santa Barbara

It has been over 20 years since Xiao-Gang Wen [1] introduced the concept of "topological order", originally to characterize fractional quantum Hall fluid phases, and more recently quantum spin liquid phases of some magnetic models (e.g. the $Z_{2}$ spin liquid in the Kagome Heisenberg antiferromagnet). Such phases have an "intrinsic topological order" and manifest a number of striking properties: Ground state degeneracies on manifolds with non-trivial topology, emergent quasiparticles with novel exchange statistics and fractional quantum numbers and, as most recently emphasized, a long-ranged entanglement in the ground state wave functions.

Despite the similarity in name, "topological insulators", predicted by Charlie Kane and others [2] and soon thereafter discovered experimentally in both two and three-dimensions, are an altogether different type of quantum phase. Topological insulators have a unique ground state on any spatial manifold, support only (unfractionalized) electron excitations, and have a short-range entangled ground state, but they are nevertheless non-trivial phases possessing exotic surface states protected by bulk symmetries. For example, the $B i_{2} S e_{3}$ and $B i_{2} T e_{3}$ class of topological insulators are protected by time-reversal invariance and electron charge conservation, and have gapless electronic surface phases consisting of a single (or odd number of) helical Dirac cones. While these surface states can be gapped by a spontaneous breaking of time-reversal symmetry on the surface or by an induced surface superconductivity, the bulk phases remain non-trivial provided these symmetries are not broken in the bulk.

It was soon realized that topological insulators represent a much broader class of "Symmetry Protected Topological" (SPT) phases [3,4] of fermions and bosons which possess distinctive surface behavior protected by bulk symmetries. For 1d and 2d SPT's the surfaces are either gapless or symmetry broken, while in 3d, gapped symmetry-respecting surfaces with (intrinsic) $2 \mathrm{~d}$ topological order are also possible.

SPT phases accessible via free Fermions were soon classified, for given bulk symmetry and spatial dimensionality [5,6]. Electron interactions (presumably) open a new realm of SPT phases, and boson and spin systems also exhibit SPT phases [4]- group cohomology has been proposed as the appropriate construction for obtaining a general classifications of spin and boson SPT phases.

Recently Lu and Vishwanath [7] presented an explicit dynamical construction of many 2d bosonic SPT phases. They employed multi-component Chern-Simons theory (so-called K-matrix theory), an approach that has been successful in describing quantum Hall states, both integer and (Abelian) fractional quantum Hall states in the Laughlin sequence and their hierarchical generalizations. 
Lu and Vishwanath introduced various global symmetries (such as time-reversal) into the multicomponent Chern-Simons approach, and specialized to the case with det $K=1$ to rule out phases with intrinsic topological order (such as FQHE states). In this way, Lu and Vishwanath were able to access many $2 \mathrm{~d}$ SPT phases with trivial bulk properties, protected by a given symmetry. As expected, these 2d SPT phases possess gapless or symmetry broken edge states.

While group cohomology classification indicates the existence of new SPT's in 3d, for bosons as well as interacting fermions, constructing explicit field theory representations for each 3d SPT, analogous to Chern-Simons theory for 2d SPT phases, remains a significant challenge.

In an important recent advance, paper (1) above, Vishwanath and Senthil (VS) initiated an attack on 3d bosonic SPT's using a "dimensionality-bootstrap" approach. When time reversal invariance is broken on the surface of an electron topological insulator, the $2 \mathrm{~d}$ surface Dirac cone can be gapped out giving a state with half-integer quantum Hall conductivity, $\sigma_{x y}= \pm 1 / 2$. If time reversal is broken with opposite signs on two halves of the surface, a $1 \mathrm{~d}$ chiral fermion (i.e. IQHE edge state) lives on the 1d boundary between the two time reversed domains. The ChalkerCoddington network model, introduced originally to describe the $2 \mathrm{~d}$ integer quantum Hall plateau transition between states with $\sigma_{x y}=0$ and $\sigma_{x y}=1$, consists of a network of $1 \mathrm{~d}$ chiral fermions which are placed to form an array of "wires" with alternating chirality. Fine tuning the inter-wire electron tunneling (to obtain an extra symmetry, time-reversal plus a translation), allows access of the gapless 2d Dirac cone.

VS have employed such a "dimensionality-bootstrap" to access the surface of some $3 \mathrm{~d}$ bosonic SPT's. VS realized that the bosonic analog of the time-reversal broken surface state of the electron topological insulator, is a state with Hall conductivity $\sigma_{x y}=1$. Domain walls between two opposite time-reversal broken regions of the surface, are taken to be the edge states of a $2 \mathrm{~d}$ bosonic IQHE phase with $\sigma_{x y}=2$. These edge states serve as the building block of a network model for the $2 \mathrm{~d}$ surface of the putative 3d bosonic SPT phase (the analog of the Chalker-Coddington network model for the electron topological insulator surface). VS argue that their network model maps to a field theory which can describe a strongly interacting critical state of bosons (similar to a "deconfined quantum point" of a $2 \mathrm{~d}$ magnet [8]). Analyzing this field theory enables VS to establish that the $2 \mathrm{~d}$ surface is either gapless, symmetry broken, or possesses intrinsic topological order. A trivial symmetry respecting surface is not possible, thereby supporting VS's claim that they have constructed the surface of a non-trivial 3d bosonic SPT.

In addition to their dimensionality-bootstrap, VS propose several bulk approaches to access 3d bosonic SPT's, including non-linear sigma models (NLSM) with topological $\theta$ - terms and B-F theory, although the precise connection between the bulk theories and the surface descriptions have yet to be elucidated. In other nice work, paper (2) above, Cenke Xu has proposed a bulk field theory description of a new $3 \mathrm{~d}$ bosonic SPT phase of an $S U(2 N)$ antiferromagnetic spin system by employing a NLSM with $\theta=2 \pi$.

It seems likely that these two new papers are only touching the tip of an iceberg in the field of 3d bosonic SPT's. And 3d interacting fermonic SPT's might be even more challenging. Progress will probably require simple model Hamiltonians which can be attacked numerically. (It is unclear whether it is possible to access 3d bosonic SPT's with sign-problem-free Hamiltonians.) It (almost!) goes without saying that such models can perhaps be engineered in cold atom systems, but one might hope that some 3d quantum magnets might exhibit SPT phases. In the realm of 3d bosonic SPT's, there is clearly much work to be done, both theoretically, (possibly) numerically, and, 
hopefully, experimentally.

\section{REFERENCES}

[1] Xiao-Gang Wen, Topological Orders in Rigid States. Int. J. Mod. Phys. B4, 239 (1990).

[2] Topological Insulators, M. Z. Hasan, C. L. Kane, Rev. Mod. Phys.82:3045 (2010).

[3] Classification of Gapped Symmetric Phases in 1D Spin Systems, Xie Chen, ZhengCheng Gu and Xiao-Gang Wen, Phys. Rev. B 83, 035107 (2011).

[4] Symmetry protected topological orders and the group cohomology of their symmetry group, Xie Chen, Zheng-Cheng Gu, Zheng-Xin Liu and Xiao-Gang Wen, arXiv:1106.4772

[5] Topological insulators and superconductors: ten-fold way and dimensional hierarchy, Shinsei Ryu, Andreas Schnyder, Akira Furusaki and Andreas Ludwig, New J. Phys. 12, $065010(2010)$

[6] Periodic table for topological insulators and superconductors, Alexei Kitaev, arXiv:0901.2686

[7] Theory and classification of interacting 'integer' topological phases in two dimensions: A Chern-Simons approach, Yuan-Ming Lu, Ashvin Vishwanath, Phys. Rev. B 86, 125119 (2012).

[8] Deconfined Quantum Critical Points by T. Senthil, A. Vishwanath, L. Balents, S, Sachdev and M.P.A. Fisher, Science, 303, 1490 (2004). 